\title{
Aspectos epidemiológicos da conidiobolomicose em ovinos na região semiárida do Nordeste do Brasil
}

\author{
Epidemiological aspects of conidiobolomycosis in sheep \\ in the Northeastern Brazilian semiarid region
}

\author{
Gildeni Maria Nascimento de Aguiar ${ }^{\mathrm{I}}$ Sara Vilar Dantas Simões ${ }^{\mathrm{I}}$ Sérgio Azevedo Santos ${ }^{\mathrm{I}}$ \\ Ana Luisa Alves Marques ${ }^{I}$ Tatiane Rodrigues da Silva ${ }^{I}$ \\ Antônio Flavio Medeiros Dantas ${ }^{\mathrm{I}}$ Franklin Riet-Correa $^{\mathrm{I}^{*}}$
}

RESUMO

Com o objetivo de determinar aspectos epidemiológicos da conidiobolomicose dos ovinos, foi realizado um estudo caso-controle em 27 propriedades (16 com casos e 11 controles) nos estados da Paraíba e do Rio Grande do Norte. Das 16 propriedades com casos, em nove (56,3\%), ocorreu apenas um foco e em sete $(43,8 \%)$ aconteceram dois, totalizando 23 focos no período do estudo. Em 20 focos, foi diagnosticada somente conidiobolomicose e, em três, além de casos de conidiobolomicose, ocorreram casos de pitiose rinofacial. Nos focos de conidiobolomicose, a morbidade variou de 0,7\% a 73,3\% e a letalidade foi de $100 \%$. Treze focos (56,5\%) aconteceram no período chuvoso (janeiro a maio), seis $(26,1 \%)$ ocorreram no período seco (junho a dezembro) e, em quatro, não foi determinada a época de ocorrência. Em todas as propriedades com casos, os animais tinham acesso a açudes, durante todo o ano ou por períodos de 2-8 meses. Na análise univariada dos fatores de risco referentes aos focos de conidiobolomicose, as variáveis associadas foram: pastejo dos animais às margens dos açudes; período de pastejo às margens dos açudes; presença de matéria vegetal constituída por plantas aquáticas; pressão de pastejo; e raça, afetando predominantemente a raça Santa Inês. Conclui-se que a associação de fatores como pastejo constante em áreas úmidas com matéria vegetal decomposta e solo rico em matéria orgânica expõe os animais à infecção por Conidiobolus spp. e predispõe à ocorrência de focos. A implantação de pastagens para corte, nas margens dos açudes, pode ser uma medida eficaz de controle da conidiobolomicose.

Palavras-chave: Conidiobolus spp., fatores de risco, ovinos, entomoftoromicose.

\section{ABSTRACT}

With the objective to determine the epidemiological aspects of conidiobolomycosis and rhinofacial pythiosis in sheep, a case-control study was conducted in 27 farms (16 cases and 11 controls) in the states of Paraíba and Rio Grande do Norte. In nine (56.3\%) farms only one outbreak was observed and in seven, two outbreaks occurred, totaling 23 outbreaks. In 20 farms only cases of conidiobolomycosis occurred and in three farms cases of conidiobolomicosis occurred together with cases of pythiosis. In conidiobolomycosis, morbidity ranged from $0.7 \%$ to $73.3 \%$ and lethality rate was $100 \%$. Thirteen outbreaks $(56.3 \%)$ occurred in the rainy season (January to May), six (26.1\%) in the dry season (June to December) and in four it was not informed. In all farms with cases, sheep had access to ponds for the whole year or for periods of 2-8 months. In the univariate analysis of risk, the variables associated with the occurrence of conidiobolomycosis were: animal grazing on the borders of the ponds; presence of vegetable matter consisting mainly of aquatic plants in the pond borders; period of grazing on the pond borders; high grazing pressure; and predominant breed of sheep, being the Santa Inês breed the most affected. It was concluded that the combination of factors such as continuous grazing in wetlands with decomposed vegetable matter and soil rich in organic matter exposes the animals to Conidiobolus spp. and predisposes to the occurrence of conidiobolomycosis. The establishment of pastures for cutting at the borders of the ponds can be an effective measure to control conidiobolomycosis.

Key words: Conidiobolus spp., risk factors, sheep, entomophthoromycosis.

\section{INTRODUÇÃO}

Rinites granulomatosas causadas por fungos da Classe Zygomycetes, incluindo Conidiobolus lamprauges (RIET-CORREA et al., 2008; DE PAULA et al., 2010; VILELA et al., 2010) ou por Pythium insidiosum, um oomiceto do Reino Straminipila (SANTURIO et al., 2008), são frequentes em ovinos. Conidiobolus spp. são fungos sapróbios, comumente encontrados em regiões tropicais,

\footnotetext{
'Hospital Veterinário, Centro de Saúde e Tecnologia Rural (CSTR), Universidade Federal de Campina Grande (UFCG), 58700-000, Patos, PB, Brasil. E-mail: rmtmed@uol.com.br. *Autor para correspondência.
} 
preferencialmente em locais úmidos, com presença de matéria vegetal em decomposição (HUMBER et al., 1989). Já $\boldsymbol{P}$. insidiosum é encontrado em águas superficiais, locais com água estagnada e, ocasionalmente, no solo (GAASTRA et al., 2010).

De acordo com CARRIGAN et al. (1992), a infecção nasal por Conidiobolus spp. ocorre pela inalação de esporos dos fungos encontrados no ambiente, não havendo transmissão da doença entre os animais. A doença pode também ser adquirida pela inoculação direta dos esporos através de microlesões nas narinas (KETTERER et al., 1992). Não tem sido estabelecida a via de infecção na pitiose rinofacial, mas, no caso da pitiose cutânea, é necessário o contato direto entre o zoósporo e a pele lesionada (GAASTRA et al., 2010).

No Brasil, em ovinos, focos de rinite causados por Conidiobolus spp. têm sido descritos no Piauí (SILVA et al., 2007a), Paraíba (RIETCORREA et al., 2008), Mato Grosso (BOABAID et al., 2008; SILVEIRA et al., 2013), Rio Grande do Sul (PEDROSO et al., 2009) e Santa Catarina (FURLAN et al., 2010). Já focos de rinite rinofacial causados por $\boldsymbol{P}$. insidiosum foram relatados no Mato Grosso (SANTURIO et al., 2008; UBIALI et al., 2013) e Paraíba (RIET-CORREA et al., 2008; PORTELA et al., 2010). Esses trabalhos descrevem, principalmente, os aspectos clínicos e patológicos da conidiobolomicose e da pitiose rinofacial, porém são escassos os dados sobre a epidemiologia dessas enfermidades. Considerando que a letalidade da conidiobolomicose tem sido de $100 \%$ (SILVA et al., 2007b) e que a letalidade da pitiose rinofacial também é muito alta (PORTELA et al., 2010), o conhecimento da epidemiologia dessas doenças pode ser a única forma de encontrar medidas de controle e profilaxia. As informações existentes mencionam a ocorrência da doença nos períodos de precipitação pluviométrica, temperatura e umidade altas, condições que permitem o melhor desenvolvimento dos agentes (SILVA et al., 2007b; BOABAID et al., 2008). Objetivou-se com este estudo compreender alguns aspectos epidemiológicos da conidiobolomicose em ovinos, incluindo raça e idade dos animais afetados e possíveis fatores ambientais associados à doença, determinando os fatores de risco, para que possam ser estabelecidas medidas adequadas de prevenção e controle.

\section{MATERIAL E MÉTODOS}

Foi elaborado um estudo do tipo casocontrole, entre março de 2009 e dezembro de 2012, em 16 propriedades com casos e 11 propriedades controle, que eram circunvizinhas e apresentavam condições de manejo semelhantes às propriedades com casos. As propriedades estavam localizadas em seis municípios do estado da Paraíba (Camalaú, São José dos Espinharas, Catingueira, Taperoá, Patos e Paulista) e três do Rio Grande do Norte (Serra Negra do Norte, Jucurutu e Caicó), todos localizados na região semiárida, caracterizada por temperatura média de $26^{\circ} \mathrm{C}$, umidade relativa de aproximadamente $50 \%$, insolação de 2800 horas ao ano, evaporação de $2000 \mathrm{~mm}^{2}$ ano $^{-1}$, chuvas de $350-800 \mathrm{~mm}^{2} \mathrm{ano}^{-1}$ e com um longo período seco, de maio-junho a fevereiromarço (MOURA et al., 2007).

Em todas as propriedades, foram aplicados questionários epidemiológicos semiestruturados, com intuito de obter informações sobre o sistema de criação, aspectos sanitários, ocorrência anterior da doença, manejo alimentar, período de pastejo nas diversas áreas da propriedade e outras informações capazes de caracterizar o ambiente dos animais no momento do foco. Com um aparelho de GPS, foram registradas as dimensões das áreas às margens dos açudes onde os animais pastejavam no período do foco.

Os diagnósticos de pitiose rinofacial ou conidiobolomicose foram realizados com base nas características clínicas (SILVA et al., 2007a; RIETCORREA et al., 2008; BOABAID et al., 2008; SANTURIO et al. 2008; PEDROSO et al., 2009; FURLAN et al., 2010; PORTELAet al., 2010; UBIALI et al., 2013). Além de constatar as características clínicas da doença, foram necropsiados 22 ovinos, nos quais foram realizados estudos histológicos. Em quatro ovinos com pitiose rinofacial e em seis com conidiobolomicose, os agentes foram identificados por imuno-histoquímica, mediante as técnicas descritas por UBIALI et al. (2013).

$\mathrm{Na}$ análise de fatores de risco, foram formados dois grupos de propriedades: propriedades com foco de rinite e propriedades sem foco. Foi conduzida uma análise univariada, em que as variáveis independentes foram cruzadas com a variável dependente (ocorrência do foco de rinite), mediante o teste de qui-quadrado ou teste exato de Fisher. Para cada variável, a categoria com menor frequência de focos foi utilizada como referência para comparação com as demais categorias. O nível de significância adotado foi de $5 \%$ e todas as análises foram realizadas com o programa SPSS for Windows, versão 9.0 (SPSS Inc., EUA).

\section{RESULTADOS}

Das 16 propriedades com casos, em nove $(56,25 \%)$, ocorreu apenas um foco e, em sete 
$(43,75 \%)$, aconteceram dois, totalizando 23 focos no período do estudo. Em 20 focos, foi feito o diagnóstico de conidiobolomicose e, em três propriedades, além de casos de conidiobolomicoses, ocorreram casos de pitiose rinofacial. Nos 20 focos de conidiobolomicose, a morbidade variou de $0,66 \%$ a $73,3 \%$ e letalidade foi de 100\%. No total, morreram 299 (22,43\%) animais, de uma população de 1333 ovinos. Em três propriedades, ocorreram casos concomitantes de conidiobolomicose e pitiose rionofacial, sendo que $17(3,55 \%)$ do total de ovinos (478) adoeceram e morreram. Desses, sete animais foram enviados para necropsia, sendo quatro diagnosticados com pitiose rinofacial e três com conidiobolomicose. Segundo os proprietários, os animais restantes apresentavam exoftalmia, sugestivo de conidiobolomicose (SILVA et al., 2007b).

Todos os casos de conidiobolomicose foram da forma rinofacial (RIET-CORREA et al., 2008). Os animais apresentavam marcada assimetria crânio-facial, muitos com exoftalmia de um olho, geralmente, com aumento de volume deste, com opacidade e ulceração da córnea e perda da visão. $\mathrm{Na}$ necropsia, observaram-se uma ou mais massas de aspecto nodular, superfície granulosa e úmida, consistência friável e coloração branca, amarela ou bronzeada, localizada no etmoide, conchas etmoidais e demais conchas nasais. Na maioria dos casos, essas massas estendiam-se para a região nasofaríngea através da coana e, em alguns, afetavam, também, os seios nasais, osso esfenoide, placa cribiforme, órbita ocular e região frontal do cérebro. Os linfonodos submandibulares e retrofaríngeos estavam geralmente aumentados de tamanho. Quatro animais tinham lesões pulmonares, um tinha lesões no fígado e um tinha envolvimento do sistema nervoso. Histologicamente, observaram-se granulomas multifocais, com áreas necróticas e eosinofílicas (material Splendore-Hoeppli), contendo hifas de 20$30 \mu \mathrm{m}$ de diâmetro, raramente septadas, semelhantes a Conidiobolus spp.

Nos casos de pitiose rinofacial, as lesões afetavam a junção mucoepitelial e pele da região anterior da face e lábio superior, causando marcado aumento de volume, que geralmente era bilateral e assimétrico. As narinas estavam parcialmente obstruídas. Nas necropsias, as lesões macroscópicas afetavam os tecidos da região vestibular do nariz, que apresentam tecido friável, de cor marrom ou amarelada. A mucosa nasal estava ulcerada e coberta por material necrótico. Ocasionalmente, a mucosa do palato duro apresentava áreas ulceradas de coloração marrom escura. Havia considerável aumento de tamanho dos linfonodos parotídeos, retrofaríngeos e submandibulares e, em dois casos, havia lesões nodulares no pulmão. Na histologia, as lesões eram semelhantes às da conidiobolomicose, porém a reação de Splendore-Hoeppli era mais discreta e as hifas mais finas $(7-10 \mu \mathrm{m})$.

$\mathrm{Na}$ análise univariada dos fatores de risco, as variáveis associadas aos focos foram: tipo de raça predominante nos rebanhos; pastejo dos animais às margens do açude; período de pastejo às margens dos açudes; presença de matéria vegetal constituída por plantas aquáticas; pressão de pastejo às margens dos açudes; e raça, afetando predominantemente a raça Santa Inês (Tabela 1).

Em 11 propriedades com casos e nas 11 controle, o sistema de manejo era extensivo e, em cinco propriedades com casos, o sistema era semiintensivo (Tabela 1). Em 22 focos, foram afetados ovinos adultos e somente em um foco foram afetados animais jovens, de menos de 18 meses (sem dentes incisivos definitivos). A condição corporal dos ovinos era satisfatória em $75 \%$ (12/16) das propriedades com foco, em $12,5 \%(2 / 16)$, os animais eram magros e, em duas, era variável.

Diferença estatística significativa $(\mathrm{P}<0,05 \%)$ foi identificada quando se comparou o padrão racial dos ovinos em propriedades com focos e controle. Das 16 propriedades com focos, em 13 $(81,25 \%)$, predominavam animais mestiços da raça Santa Inês e, em três $(18,75 \%)$, os rebanhos eram formados por animais sem padrão racial definido. Dentre as 11 propriedades controle, em três $(27,28 \%)$, os animais eram mestiços da raça Santa Inês e, em oito $(72,72 \%)$, os animais não tinham padrão racial definido (Tabela 1).

Em relação ao período de ocorrência, 13 dos 23 focos $(56,52 \%)$ aconteceram no período chuvoso (entre janeiro e maio) e seis $(26,08 \%)$ ocorreram no período seco (junho a dezembro). Em quatro propriedades com mais de um foco, os proprietários não conseguiram informar com precisão se, na época em que ocorreram alguns dos focos, ainda estava chovendo ou já tinha iniciado a estação seca (Tabela 1).

Todas as propriedades, casos ou controles, possuíam açudes. Em $75 \%$ (12/16) das propriedades com casos, os animais tinham acesso durante todo o ano a essas áreas úmidas e, em 25\% (4/16), o acesso ocorria durante dois a oito meses do ano. $\mathrm{O}$ acesso durante todo o ano foi registrado em apenas 45,5\% (5/11) das propriedades controle. Em 27,27\% (3/11) das propriedades controle, o acesso ocorria somente durante dois a oito meses do ano e, em três propriedades, os animais não pastejavam nas margens de açudes. $\mathrm{O}$ 
Tabela 1 - Fatores de risco associados à conidiobolomicose em ovinos na região semiárida do Brasil, no período de 2009 a 2012.

\begin{tabular}{|c|c|c|c|}
\hline Variável & № total de Propriedades & $\mathrm{N}^{\mathrm{o}}$ propriedades com focos & $\mathrm{P}$ \\
\hline \multicolumn{4}{|l|}{ Raça Predominante } \\
\hline Sem Padrão Racial Definido & 11 & $3(27,27 \%)$ & \multirow{2}{*}{0,015} \\
\hline Santa Inês & 16 & $13(81,25 \%)$ & \\
\hline \multicolumn{4}{|l|}{ Sistema de manejo } \\
\hline Extensivo & 22 & $11(50 \%)$ & \multirow{2}{*}{0,06} \\
\hline Semi-intensivo & 5 & $5(100 \%)$ & \\
\hline \multicolumn{4}{|l|}{ Pastejo nas margens do açude } \\
\hline Sim & 24 & $16(66,66 \%)$ & \multirow{2}{*}{0,05} \\
\hline Não & 3 & 0 & \\
\hline \multicolumn{4}{|l|}{ Período de pastejo às margens do açude } \\
\hline Sempre & 17 & $12(73,33 \%)$ & 0,049 \\
\hline 2-8 meses & 7 & $3(66,66 \%)$ & 0,200 \\
\hline Não pastejavam & 3 & 0 & $*$ \\
\hline \multicolumn{4}{|l|}{ Matéria vegetal às margens do açude } \\
\hline $\operatorname{Sim}$ & 22 & $16(72,72 \%)$ & \multirow{2}{*}{0,05} \\
\hline Não & 5 & 0 & \\
\hline \multicolumn{4}{|l|}{ Perímetro do açude } \\
\hline até $0,442 \mathrm{~km}$ & 6 & $3(50 \%)$ & $*$ \\
\hline $0,443-1,237 \mathrm{~km}$ & 11 & $7(63,6 \%)$ & 0,644 \\
\hline$>1,237 \mathrm{~km}$ & 6 & $5(83,3 \%)$ & 0,546 \\
\hline Sem acesso a açude & 4 & 0 & 0 \\
\hline \multicolumn{4}{|l|}{ Pressão de pastejo } \\
\hline Alta & 17 & $15(88,23 \%)$ & 0,0003 \\
\hline Baixa & 5 & $1(20 \%)$ & 0,028 \\
\hline Não pastejavam ou não havia forragem disponível & 5 & 0 & $*$ \\
\hline
\end{tabular}

número de propriedades que os animais pastejavam sempre em áreas com açude foi significativamente maior $(\mathrm{P}<0,05)$ nas propriedades com foco do que nas propriedades controle (Tabela 1 ).

A parte do perímetro dos açudes disponibilizada para pastejo dos animais era variável entre as propriedades, alcançando de 0,265 a dois quilômetros (Tabela 1). Não foi constatada diferença estatística entre este perímetro e a ocorrência dos focos, porém houve uma tendência dos focos ocorrerem nas propriedades em que o perímetro era maior. Em oito das propriedades com focos, existiam açudes de maior capacidade hídrica. Quatro dessas propriedades possuíam os maiores perímetros e $80,69 \%$ (255/316) das mortes ocorreram nessas propriedades.

Nas propriedades com focos, nas margens dos açudes predominavam plantas aquáticas como Eichornia crassipes, conhecida como "pasta", Ludwigia helminthorrhiza, denominada "crote", e Nymphaea sp., chamada de "goife". Ocasionalmente, havia pouca quantidade de plantas forrageiras, como Brachiaria spp., em quantidade inferior às plantas aquáticas, sendo observadas apenas pequenas touceiras. Em 88,23\% (15/17) dessas propriedades, foi observada também alta pressão de pastejo nas margens do açude, o que diferiu estatisticamente $(\mathrm{P}<0,05)$ das propriedades controle (Tabela 1$)$. Havia relatos de animais que pastejavam muito próximo às margens dos açudes ou mesmo entravam na água para ingerir plantas aquáticas. Em 54,54\% (6/11) das propriedades controle, nas que a pressão de pastejo foi significativamente menor, havia plantas forrageiras em maior proporção do que plantas aquáticas nas margens dos açudes. Em duas dessas, a margem do açude era dividida, de modo que os animais passavam dois meses em cada subdivisão, com o intuito de permitir que a Brachiaria spp. plantada as suas margens pudesse atingir uma boa altura de pastejo, não prejudicando o crescimento da forrageira nos próximos períodos. Em uma propriedade, embora a área não fosse dividida, os animais passavam apenas dois meses pastejando na margem do açude.

\section{DISCUSSÃO}

A observação de 23 focos em que ocorreram casos de conidiobolomicose e de somente três em que foram observados casos de pitiose rinofacial, 
simultaneamente aos de conidiobolomicose, sugere que, na região semiárida da Paraíba e Rio Grande do Norte, a conidiobolomicose é bem mais frequente do que a pitiose rinofacial. Resultados semelhantes foram descritos por PORTELA et al. (2010), que, entre 2003 e 2009, na Paraíba, descreveram dois focos de pitiose rinofacial com casos esporádicos e sete focos de conidioblomicose, com morbidade de $1,25 \%$ a $20 \%$ e letalidade de $100 \%$. Em 25 focos de conidiobolomicose descritos por SILVA et al. (2007b), no Piauí, a morbidade média anual, em 25 propriedades, variou de $2,4 \%$ a $3,4 \%$, enquanto que a morbidade por propriedade variou de $0,1 \%$ a $14,3 \%$, com uma letalidade de $100 \%$. Esses dados, no seu conjunto, demostram a importância da conidiobolomicose em ovinos no semiárido, o que, associado à ineficiência do tratamento, evidencia a necessidade de conhecer aspectos epidemiológicos da doença para determinar medidas de controle.

A ocorrência de focos com alta morbidade demonstra que os animais estão sendo muito expostos aos agentes. HUMBER et al. (1989) ressaltaram que as micoses causadas por fungos sapróbios não são facilmente adquiridas e que os hospedeiros precisam ser constantemente desafiados ou estar imunologicamente comprometidos para que a infecção ocorra. O pastejo nas áreas dos açudes onde há umidade, detritos de plantas e solo rico em matéria orgânica, seguramente expõe os animais aos agentes e favorece a ocorrência da enfermidade. Por outro lado, o estado nutricional satisfatório da maioria dos animais acometidos sugere que, em ovinos, fatores que conduzam a uma queda na imunidade não são necessários para o desenvolvimento da conidiobolomicose. KETTERER et al. (1992) sugerem, também, que não é necessário depressão imunológica para a ocorrência da conidiobolomicose. Estudos em humanos são controversos quanto à necessidade de uma queda no estado imunológico e a ocorrência da conidiobolomicose, pois a doença acomete tanto pacientes saudáveis como aqueles com imunossupressão transitória (RIBES et al., 2000).

Em relação ao maior número de casos de conidiobolomicose em propriedades que criavam animais da raça Santa Inês, quando comparados a animais sem raça definida, seria precipitado associar a ocorrência das enfermidades a uma determinada raça, pois, na literatura, não existem informações sobre a influência racial e predisposição às infecções fúngicas em ovinos. Além disso, apesar de no Brasil o maior número de relatos da conidiobolomicose ter acometido animais da raça Santa Inês e seus mestiços (RIET-CORREA et al., 2008; BOABAID et al., 2008;
FURLAN et al., 2010; UBIALI et al., 2013), deve-se considerar que essa é uma das principais raças criadas no país, sendo grande a possibilidade de um animal dessa raça vir a ser acometido. No Rio Grande do Sul, a enfermidade foi diagnosticada em ovinos da raça Texel (PEDROSO et al., 2009) e, no Piauí, além da Santa Inês, animais da raça Morada Nova foram acometidos (SILVA et al., 2007b).

Em relação à idade dos animais afetados, o fato de que somente em um foco ocorreram casos em animais de até 18 meses sugere que os animais adultos são mais susceptíveis. No entanto, não foram realizados testes estatísticos porque, na maioria dos casos, não foi informado o número de ovinos jovens e adultos que constituíam o rebanho.

No estado do Piauí (SILVA et al., 2007b), focos frequentes e recorrentes de conidiobolomicose são associados a períodos de alta precipitação pluviométrica, enquanto que, neste estudo, foi observado que há registros de focos também no período seco, demonstrando que outros fatores, além da precipitação pluviométrica, contribuem para o surgimento dos casos. Em Mato Grosso e Santa Catarina (BOABAID et al., 2008; FURLAN et al., 2010), os focos foram também associados ao período chuvoso ou ao final das chuvas.

Neste trabalho, os fatores de risco associados aos focos das rinites incluíram o pastejo dos animais às margens do açude, a presença de matéria vegetal abundante constituída por plantas aquáticas e a pressão de pastejo, sugerindo que as condições ambientais em áreas ao redor dos açudes são determinantes para a ocorrência da doença. A ocorrência de focos mesmo após o final do período de chuvas é atribuída à presença de matéria vegetal decomposta das plantas aquáticas presentes à margem dos açudes, pois altos índices de umidade e altas temperaturas favorecem o desenvolvimento de fungos. Nas propriedades onde havia mortalidade dos animais no período seco, as margens das aguadas eram uma das principais fontes de forragem verde, $o$ que favorecia e intensificava o pastejo nessas áreas, aumentando a exposição dos animais aos agentes. Em outros trabalhos, a presença de matéria orgânica nos locais de pastejo, associada à umidade, foram também relacionadas à ocorrência de focos (CARRIGAN et al., 1992; SILVA et al., 2007a; 2007b; BOABAID et al., 2008; RIET-CORREA et al., 2008).

Apesar de plantas aquáticas margeando os açudes terem sido também observadas em propriedades controle, as possibilidades da ocorrência da doença estão aparentemente diminuídas por: plantio de forrageiras de porte mais cespitoso, a exemplo de 
Brachiaria spp.; utilização das áreas ao redor dos açudes de forma rotacionada; e redução do tempo de permanência dos animais nessas áreas, diminuindo a pressão de pastejo e evitando o pastoreio rente ao solo e a inalação do fungo.

A tendência dos focos ocorrerem com maior frequência nas propriedades que possuíam açudes de maior perímetro, e o maior número de óbitos observados nas propriedades com reservatórios mais volumosos podem estar associados ao fato de que, por mais que haja uma redução no volume dos açudes, esses não secam e os animais têm acesso a áreas úmidas tanto no período seco quanto no chuvoso. Essas áreas úmidas são denominadas de vazantes e possuem solo fértil e úmido, facilitando o desenvolvimento de plantas e formamdo um ambiente favorável para a proliferação de fungos.

A análise dos resultados obtidos permitiu esclarecer aspectos epidemiológicos importantes da conidiobolomicose na região semiárida e sugere que é possível minimizar ou mesmo evitar a ocorrência desta com medidas relativamente fáceis de serem implantadas, tais como: a redução da pressão de pastejo nas margens dos açudes; a implantação de pastagens; e a divisão do perímetro dos açudes em áreas que possam ser utilizadas de forma rotacionada. Outra opção seria impedir o acesso dos animais aos açudes e utilizar as vazantes exclusivamente como áreas para produção de forragens de corte.

\section{CONCLUSÃO}

A conidiobolomicose é mais frequente do que a pitiose rinofacial no semiárido da Paraíba e do Rio Grande do Norte. A alta frequência da conidiobolomicose está associada ao pastejo dos ovinos em áreas nas margens de açudes, com presença de plantas aquáticas e alta pressão de pastejo. Algumas medidas como a implantação de forrageiras para corte, nas beiras dos açudes, podem ser eficientes no controle da enfermidade.

\section{REFERÊNCIAS}

BOABAID, F.M. et al. Conidiobolomicose em ovinos no Estado de Mato Grosso. Pesquisa Veterinária Brasileira, v.28, n.1, p.77-81, 2008. Disponível em: <http://dx.doi.org/10.1590/S0100736X2008000100012>. Acesso em: 23 jul. 2009.

CARRIGAN, M.J. et al. Ovine nasal zygomycosis caused by Conidiobolus incongruus. Australian Veterinary Journal, v.69, n.10, p.237-240, 1992. Disponível em: <http:// doi/10.1111/j.1751-0813.1992.tb09869.x/pdf>. Acesso: 09 mar. 2011.

DE PAULA, D.A.J. et al. Molecular characterization of ovine zigomycosis in central western Brazil. Journal of Veterinary
Diagnostic Investigation, v.22, n.2, p.274-277, 2010. Disponível em: <http://vdi.sagepub.com/content/22/2/274.full.pdf+html>. Acesso em: 11 mar. 2011. doi:10.1177/104063871002200220.

FURLAN, F.H. et al. Conidiobolomicose causada por Conidiobolus lamprauges em ovinos no Estado de Santa Catarina. Pesquisa Veterinária Brasileira, v.30, n.7, p.529-532, 2010. Disponível em: <http://dx.doi.org/10.1590/S0100-736X2010000700003〉. Acesso em: 11 mar. 2011.

GAASTRA, W. et al. Pythium insidiosum: an overview. Veterinary Microbiology, v.146, p.1-16, 2010. Disponível em: <http://dx.doi. org/10.1016/j.vetmic.2010.07.019>. Acesso em: 11 mar. 2011.

HUMBER, R.A. et al. Equine zygomycosis caused by Conidiobolus lamprauges. Journal of Clinical Microbiology, v.27, n.3, p.573-576, 1989. Disponível em: <http://jcm.asm.org/ content/27/3/573.full.pdf >. Acesso em: 11 mar. 2011.

KETTERER, P.J. et al. Rhinocerebral and nasal zygomycosis caused by Conidiobolus incongruus. Australian Veterinary Journal, v.69, n.4, p.85-87, 1992. Disponível em: <http://doi/10.1111/j.1751-0813.1992. tb15556.x/pdf>. Acesso em: 09 mar. 2011.

MOURA, M.S.B. et al. Clima e água de chuva no semiárido. Disponível em: <http://ainfo.cnptia.embrapa.br/digital/bitstream/ CPATSA/36534/1/OPB1515.pdf>. Acesso em: 02 out. 2013.

PEDROSO, P.M.O. et al. Rinite micótica rinofaríngea em um ovino Texel no Rio Grande do Sul. Acta Scientiae Veterinariae, v.37, n.2, p.181-185, 2009. Disponível em: <http://www.lume.ufrgs. $\mathrm{br} / \mathrm{bitstream} /$ handle/10183/20886/000729471.pdf?sequence $=1>$. Acesso em: 29 jul. 2011.

PORTELA, R.A. et al. Doenças da cavidade nasal de ruminantes no Brasil. Pesquisa Veterinária Brasileira, v.30, n.10, p.884854, 2010. Disponível em: <http://dx.doi.org/10.1590/S0100736X2010001000007>. Acesso em: 02 nov. 2013.

RIBES, J.A. et al. Zygomycetes in human disease. Clinical Microbiology Reviews, v.13, n.2, p.236-301, 2000. Disponível em: <http://cmr.asm.org/content/13/2/236.full.pdf+html>. Acesso em: 29 jul. 2011.

RIET-CORREA, F. et al. Outbreaks of rhinofacial and rhinopharygeal zygomycosis in sheep in Paraíba, northeastern Brazil. Pesquisa Veterinária Brasileira, v.28, n.1, p.2935, 2008. Disponível em: <http://dx.doi.org/10.1590/S0100736X2008000100005>. Acesso em: 23 jul. 2009.

SANTURIO, J.M. et al. Granulomatous rhinitis associated with Pythium insidiosum infection in sheep. Veterinary Record, v.164, n.9, p.276-277, 2008. Disponível em: <http://doi:10.1136/ vr.163.9.276>. Acesso em: 27 jul. 2011.

SILVA, S.M.M. et al. Conidiobolomycosis in sheep in Brazil. Veterinary Pathology, v.44, p.314-319, 2007a. Disponível em: <http://vet.sagepub.com/content/44/3/314〉. Acesso em: 23 jul. 2009.

SILVA, S.M.M. et al. Epidemiologia e sinais clínicos da conidiobolomicose em ovinos no Estado do Piauí. Pesquisa Veterinária Brasileira, v.27, n.4, p.184-190, 2007b. Disponível em: <http://dx.doi.org/10.1590/S0100-736X2007000400010>. Acesso em: 23 jul. 2009. 
SILVEIRA, M.M. et al. Development and application of polymerase chain reaction test for detection of Conidiobolus lamprauges. Pesquisa Veterinária Brasileira, v.33, n.12, p.14481452, 2013. Disponível em: <http://dx.doi.org/10.1590/S0100736X2013001200009>. Acesso em: 23 fev. 2014.

UBIALI, D.G. et al. Pathology of nasal infection caused by Conidiobolus lamprauges and Pythium insidiosum in sheep.
Journal of Comparative Pathology, v.149, n.2/3, p.137-145, 2013. Disponível em: <http://dx.doi.org/10.1016/j.jcpa.2012.12.002〉. Acesso em: 13 out. 2012.

VILELA, R. et al. Morphologic and phylogenetic characterization of Conidiobolus lamprauges recovered from infected sheep. Journal of Clinical Microbiology, v.48, n.2, p.427-432, 2010. Disponível em: 〈http://doi:10.1128/JCM.01589-09〉. Acesso em: 02 nov. 2013.

Ciência Rural, v.44, n.12, dez, 2014. 